\title{
Establishment of In Vitro Brain Models for AON Delivery
}

\section{Elena Daoutsali and Ronald A. M. Buijsen}

\begin{abstract}
Progress in stem cell biology has made it possible to generate human-induced pluripotent stem cells (hiPSC) that can be differentiated into complex, three-dimensional structures, where the cells are spatially organized. To study brain development, Lancaster and colleagues developed an hiPSC-derived threedimensional organoid culture system, termed cerebral organoids, that develop various discrete, although interdependent, brain regions. Here we describe in detail the generation of cerebral organoids using a modified version of the culture protocol.
\end{abstract}

Key words Cerebral organoid, Disease modeling, Induced pluripotent stem cells

\section{Introduction}

Many brain disorders are hereditary diseases with a known genetic cause, which allowed scientists to generate animal models to study disease progression, understand disease mechanisms, and perform therapeutic intervention studies $[1,2]$. However, (1) mice are different from humans, and it is difficult to translate results from animal experiments into clinical application; (2) the genetic cause of many diseases is not yet known; (3) many disease-causing genes are mainly expressed in the cells that are affected; (4) for many of them, there are no (humanized-)mouse models available; (5) there is governmental and public pressure to advance the development of alternative model systems to replace animal studies. This emphasizes the need for patient-derived disease models that bridge the translational gap between animal models and human clinical trials. Progress in stem cell biology has made it possible to generate human induced pluripotent stem cells (hiPSCs) [3] that can be differentiated into the important cell types of the brain, neurons, and astrocytes $[4,5]$. The disadvantage of these $2 \mathrm{D}$ models is that they are descriptive at a cellular level, but they fail to adequately provide the details that could be derived from a more complex, three-dimensional structure, where the cells are spatially organized 
[6]. In 2013, Lancaster and colleagues developed a hiPSC-derived three-dimensional organoid culture system, termed cerebral organoids, that develop various discrete, although interdependent, brain regions [7]. These organoids recapitulate many features of human cortical development, including a progenitor zone organization with abundant outer radial glial stem cells [8].

Here we describe the generation of cerebral organoids using a modified version of the Lancaster protocol $[7,9]$. In short, feederfree cultured hiPSCs were dissociated and replated in neural induction medium in a non-adherent cell culture plate, and differentiated for 100 days (Fig. 1). Cryosections of these organoids can be used for immunofluorescence studies. Organoids can be used for many different purposes including disease modeling, studying disease mechanisms, or analyzing therapeutic interventions (using for example antisense oligonucleotides) at any given time point.

\section{Materials}

\subsection{Neuroectodermal Differentiation}

2.2 Neurospheres
1. $\mathrm{mTeSR}^{\mathrm{TM}} 1$.

2. Matrigel.

3. Dulbecco's Modified Eagle's Medium (DMEM) and Ham's F-12 Nutrient Mixture (DMEM/F12).

4. ACCUTASETM.

5. STEMdiff' ${ }^{\text {TM }}$ Neural Induction Medium (NIM).

6. Y-27632.

7. v-bottom shape 96-well plate.

1. Neurosphere medium: DMEM/F12 and Neurobasal medium 1:1, 1:200 N2 supplement, 1:100 B-27 supplement (without vitamin $\mathrm{A}$ ), 1:100 L-glutamine, $0.05 \mathrm{mM}$ non-essential amino acids (MEM-NEAA), $100 \mathrm{U} / \mathrm{ml}$ penicillin, $100 \mu \mathrm{g} / \mathrm{ml}$ streptomycin, $1.6 \mathrm{mg} / \mathrm{l}$ insulin, $0.05 \mathrm{mM} \beta$-mercaptoethanol.

2. Wide orifice pipette tips.

3. Organoid embedding sheet (or parafilm and a $200 \mu \mathrm{l}$ tip box).

1. Brain organoid medium: DMEM/F12 and Neurobasal medium 1:1, 1:200 $\mathrm{N}_{2}$ supplement, 1:100 B27 supplement w/o vitamin A, 1:100 L-glutamine, $0.05 \mathrm{mM}$ MEM-NEAA, $100 \mathrm{U} / \mathrm{ml}$ penicillin, $100 \mu \mathrm{g} / \mathrm{ml}$ streptomycin, $1.6 \mathrm{mg} / \mathrm{l}$ insulin, $0.5 \mu \mathrm{M}$ dorsomorphin, $5 \mu \mathrm{M}$ SB431542, $0.05 \mathrm{mM}$ $\beta$-mercaptoethanol.

2. Spinner flask or 6-well plates.

3 . Bioreactor or shaker. 


\begin{tabular}{|c|c|c|c|c|c|}
\hline \multicolumn{2}{|c|}{ Initiation of neuroectoderm } & $\begin{array}{c}\text { Neurosphere } \\
\text { formation }\end{array}$ & \multicolumn{3}{c|}{ Cerebral organoid maturation } \\
\hline Day 3 & Day 5 & Day 10 & Day 16 & Day 23 & Day 40 \\
\hline & & & & & \\
\hline
\end{tabular}

Fig. 1 Cerebral organoids during the various stages of organoid culturing. Organoids are cultured using a modified version of the Lancaster protocol. After 5 days of neuroectodermal differentiation, the neurospheres are embedded in Matrigel and cultured in the neurosphere medium in a 6-well plate for 5 days. For cerebral organoid maturation, the embedded neurospheres are transferred into a spinner flask and can be used for downstream applications if needed

\subsection{Fixation and Embedding}

2.5 Cryosectioning
and Immunofluorescent Staining
1. Wide orifice pipette tips.

2. Dulbecco's Phosphate Buffered Saline (DPBS).

3. $4 \%$ paraformaldehyde (PFA).

4. $30 \%$ sucrose in distilled water.

5. Embedding mold.

6. Optimum cutting temperature compound (OCT).

1. PLL-coated glass cryoslides.

2. Barrier pen.

3. PBS-glycine: $200 \mathrm{mM}$ of glycine in DPBS.

4. Blocking Solution: $5 \%$ goat or horse serum, $0.1 \%$ Triton $\mathrm{X}-100,200 \mathrm{mM}$ glycine in Dulbecco's Phosphate-Buffered Saline.

5. Immunobuffer solution: $1 \%$ goat or horse serum, $0.1 \%$ Triton X-100 in Dulbecco's Phosphate-Buffered Saline.

6. Prolong Diamond Antifade Mounting (+DAPI).

\section{Methods}

\subsection{Neuroectodermal Differentiation}

1. Culture hiPSCs under feeder-free conditions in $\mathrm{mTeSR}^{\mathrm{TM}} \mathrm{l}$ in a culture dish coated with Matrigel. For neuroectodermal differentiation one $100 \mathrm{~mm}$ cell culture dish is required (see Note $\mathbf{1}$ ).

2. When the hiPSCs are ready for passaging, wash the hiPSCs with $10 \mathrm{ml}$ pre-warmed $\left(37^{\circ} \mathrm{C}\right)$ DMEM/F12.

3. Remove DMEM/F12, add $2 \mathrm{ml}$ pre-warmed ACCUTASETM and incubate for $5 \mathrm{~min}$ at $37^{\circ} \mathrm{C}$ and $5 \% \mathrm{CO}_{2}$, allowing cells to detach (see Note 2). 


\subsection{Neurosphere Embedding}

4. Pipette the cell suspension up and down 3-5 times using a $1-\mathrm{ml}$ micropipette to make a single-cell suspension and collect the suspension in a $15-\mathrm{ml}$ tube (see Note 3).

5. Add $6 \mathrm{ml}$ of pre-warmed DMEM/F12, wash the culture dish and collect the suspension in the same $15-\mathrm{ml}$ tube as in step 4.

6. Centrifuge at $300 \times g$ for $5 \mathrm{~min}$ at room temperature.

7. Remove supernatant and resuspend the cell pellet in $1 \mathrm{ml}$ of NIM supplemented with $10 \mu \mathrm{M}$ Y-27632 and count the cells using a cell counter.

8. Dilute the cell suspension in NIM supplemented with $10 \mu \mathrm{M}$ Y-27632 to $4.5 \times 10^{5}$ cells per $\mathrm{ml}$ and add $100 \mu \mathrm{l}$ per well in a non-adherent, v-bottom shape, 96-well plate (see Note 4).

9. Centrifuge the plate at $500 \times \mathfrak{g}$ for $3 \mathrm{~min}$ at room temperature and incubate at $37^{\circ} \mathrm{C}$ and $5 \% \mathrm{CO}_{2}$.

10. Change medium by carefully removing $50 \mu \mathrm{l}$ NIM from the top of the wells, without disturbing the embryoid bodies, and by adding $50 \mu \mathrm{l}$ of fresh NIM. Medium changes should be done daily for the next 5 days (see Note 5 ).

1. Use an 1-ml micropipette with a wide orifice pipet tip to place the neurospheres on a silicone organoid embedding sheet (see Notes 6 and 7).

2. Carefully remove the medium from the well, without disturbing the neurosphere, and expel it back to dislodge the neurosphere from the bottom of the well.

3. Use a wide orifice tip to collect the neurosphere and transfer it to the embedding sheet.

4. Carefully remove as much liquid from the embedding sheet as possible.

5. Use an ice-cold tip to add a drop of Matrigel onto each neurosphere.

6. Use an ice-cold tip to place the neurosphere in the center of the Matrigel droplet.

7. Incubate at $37^{\circ} \mathrm{C}$ and $5 \% \mathrm{CO}_{2}$ for $15 \mathrm{~min}$.

8. Carefully wash the neurospheres from the embedding sheet into a $100-\mathrm{mm}$ culture dish by flushing them with the neurosphere medium and incubate at $37{ }^{\circ} \mathrm{C}$ and $5 \% \mathrm{CO}_{2}$. The total volume of neurosphere medium in the dish is $10 \mathrm{ml}$ (see Note 8 ).

9. Add $2 \mathrm{ml}$ of fresh neurosphere medium on day 2 .

10. Use the embedded neurospheres 4 days after the embedding for the next step in the protocol. 


\subsection{Organoids}

\subsection{Fixation and Embedding}

1. Carefully transfer the embedded neurospheres into a spinner flask containing $100 \mathrm{ml}$ of pre-warmed organoid medium using a 2-ml serological pipette (see Note 9).

2. Place the spinner flask on the magnetic stirring platform in the incubator and use a stirring program at $25 \mathrm{rpm}$.

3 . Culture the organoids on the magnetic stirring platform at $37{ }^{\circ} \mathrm{C}$ and $5 \% \mathrm{CO}_{2}$ for up to 100 days (see Notes 10 and 11 ).

4. Change the medium weekly by removing $50 \mathrm{ml}$ of the organoid medium and adding $50 \mathrm{ml}$ of fresh organoid medium (see Note 12). To refresh the medium, remove the spinner flask and let the organoids sink to the bottom for $5 \mathrm{~min}$. Then carefully remove the medium, without disturbing the organoids.

1. Collect the organoids with an 1-ml micropipette with a wide orifice pipet tip and transfer them in a $60-\mathrm{mm}$ dish or in a 6-well plate ( see Note 6).

2. Wash the organoids with $5 \mathrm{ml}$ of prewarmed Dulbecco's Phosphate-Buffered Saline.

3. Use a wide orifice tip to transfer each organoid separately into a 1.5-ml Eppendorf tube with $500 \mu \mathrm{l}$ of $4 \%$ PFA. Incubate the organoids for $30 \mathrm{~min}$ at room temperature (see Notes 13 and 14).

4. Remove the 4\% PFA solution and wash the organoids twice with $1 \mathrm{ml} \mathrm{DPBS}$ for $5 \mathrm{~min}$ ( see Note 15).

5. Remove DPBS and add $1 \mathrm{ml}$ of $30 \%$ sucrose in distilled water per tube to dehydrate the organoids and incubate the organoids at $4{ }^{\circ} \mathrm{C}$ overnight ( see Note 16).

6. Fill a Peel-A-Way embedding molds with $400 \mu \mathrm{l}$ of optimum cutting temperature compound (until the middle) and use an inoculation loop to place the organoid in the center of the mold. Label the rim of the mold with the sample name (see Note 17).

7. Snap-freeze the organoid-containing mold with ethanol on dry-ice and store at $-80^{\circ} \mathrm{C}$ until further use.

1. Section cryoprotected frozen organoids into 16- to $20-\mu \mathrm{m}$ thick slices on PLL-coated glass cryoslides using a cryostat (see Notes 18 and 19).

2. Thaw (if they were frozen) and dry the slides for $30 \mathrm{~min}$ at room temperature.

3. Draw a hydrophobic barrier around each section using a barrier pen.

4. To quench the PFA-induced autofluorescence wash the slides twice with $200 \mu \mathrm{L}$ of PBS-glycine for $3 \mathrm{~min}$. 

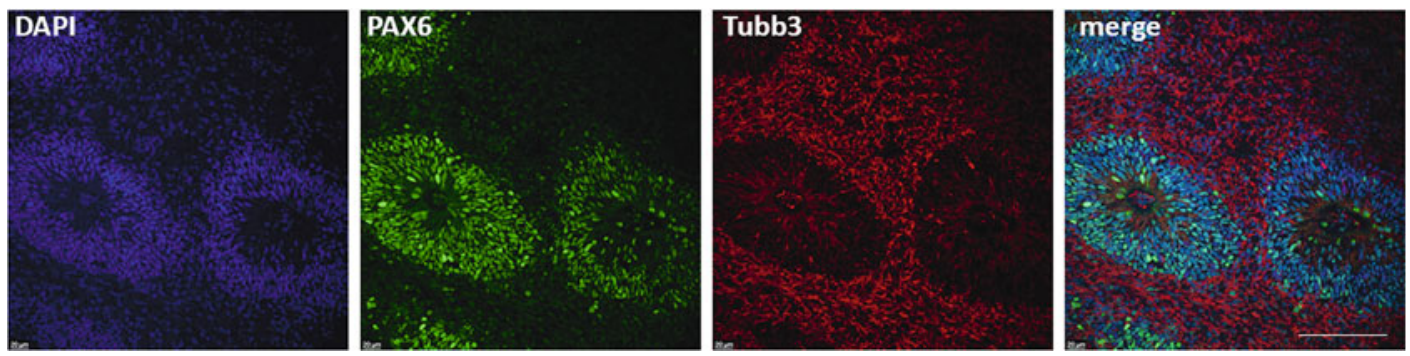

Fig. 2 Immunofluorescent staining of a cortical plate structure. Cortical plate structure in cerebral organoids stained with DAPI (blue), the neural progenitor marker PAX6 (green), and the neural marker TUBB3 (red). The scale bar represents $100 \mu \mathrm{m}$

5. Block nonspecific binding by adding $100 \mu \mathrm{l}$ of Blocking Solution to the section for $1 \mathrm{~h}$ at room temperature.

6. Add $100 \mu \mathrm{l}$ of primary antibody diluted in immunobuffer solution and incubate the slides overnight at $4{ }^{\circ} \mathrm{C}$ (see Note 20).

7. Wash three times with DPBS for $5 \mathrm{~min}$.

8 . Incubate the sections with the secondary antibody in immunobuffer solution for 1.5-2 h at room temperature (see Note 21).

9. Wash the slides three times in PBS.

10. Put a drop of Prolong Diamond Antifade Mountant (+DAPI) on the section and put a coverslip on top. Leave overnight at room temperature.

11. Store in fridge at $4{ }^{\circ} \mathrm{C}$ until performing microscopy. An example of a cortical plate structure in cerebral organoids stained with the neural progenitor marker PAX6 and the neural marker TUBB3 can be seen in Fig. 2.

\section{Notes}

1. Change $\mathrm{mTeSR}^{\mathrm{TM}} 1$ daily and passage hiPSCs after $5-7$ days. hiPSCs are ready to passage when the majority of the colonies are large, compact, and have centers that are dense compared to their edges. Only use undifferentiated, high-quality hiPSCs that are fully characterized according to the latest human pluripotent stem cell registry guidelines (https://hpscreg.eu/).

2. $1 \mathrm{ml}$ ACCUTASETM per $25 \mathrm{~cm}^{2}$ surface area.

3. ACCUTASETM is a cell detachment solution of proteolytic and collagenolytic enzymes and does not need to be neutralized.

4. Make sure that the cells are equally distributed in the suspension by mixing the tube regularly. 
5. There is more evaporation from the four corner wells. Do not use these wells or add extra medium daily (up to $100 \mu$ l total culture volume).

6. The wide orifice tip can be replaced by a cut tip. The cut on the tip should be done by using sterile scissors. You can effectively sterilize scissors in an autoclave, but tools can also be sterilized in alcohol or a flame.

7. The silicone organoid embedding sheet can be replaced by placing parafilm over an empty $200-\mu$ tip box and using a finger to make small holes.

8. A maximum of 20 neurospheres per $100-\mathrm{mm}$ cell culture dish.

9. A maximum of 20 organoids per culture flask.

10. When spinner flasks are not available, non-adhesive culture plates on a rotating platform can be used. Use a shaking program of $75 \mathrm{rpm}$.

11. If an orbital shaker and a 6-well plate are used, do not transfer more than 10 organoids per well. Moreover, monitor the organoids regularly to reduce the chance of them sticking together. If organoids stick together, you can easily separate these by using a sterile pipet tip as a knife.

12. Medium changes need to be done more often when there is a color change of the medium. Check the organoids regularly and replace the medium as done in step 3 in Subheading 3.3 when needed. For a 6-well plate, a volume of 3-ml medium per well can be used and medium should be changed twice a week and/or when there is a color change of the medium.

13. For a big batch of organoids, fixation can be done in a 6-well plate.

14. For larger organoids $(>2 \mathrm{~mm})$, a $4 \%$ PFA incubation at $4{ }^{\circ} \mathrm{C}$ overnight is recommended.

15. You can store the organoids in $1 \mathrm{ml} \mathrm{DPBS}$ at $4{ }^{\circ} \mathrm{C}$ for up to 7 days.

16. After the addition of $30 \%$ sucrose solution, the organoids should float at the surface, and by the next day, the organoids should sink down to the bottom of the tube. You can keep the organoid in $30 \%$ sucrose for up to a month. The recommended time for organoids larger than $4 \mathrm{~mm}$ is $5-7$ days.

17. To better visualize the organoid while cryosectioning, add Trypan-blue diluted 1:50 in DPBS before embedding the organoids in OCT (and after 30\% sucrose) for $15 \mathrm{~min}$ at room temperature. The outer area of the organoid will be colored blue.

18. Up to 100 sections can be obtained from one organoid.

19. The slides can be stored at $-80^{\circ} \mathrm{C}$. 
20. Put a wet tissue inside the box to prevent the slides from drying out.

21. Keep in the dark from here on.

\section{References}

1. Chesselet MF, Carmichael ST (2012) Animal models of neurological disorders. Neurotherapeutics 9(2):241-244. https://doi.org/10. 1007/s13311-012-0118-9

2. Berman RF, Buijsen RA, Usdin K, Pintado E, Kooy F, Pretto D, Pessah IN, Nelson DL, Zalewski Z, Charlet-Bergeurand N, Willemsen R, Hukema RK (2014) Mouse models of the fragile $\mathrm{X}$ premutation and fragile $\mathrm{X}$-associated tremor/ataxia syndrome. J Neurodev Disord 6(1):25. https://doi.org/10.1186/ 1866-1955-6-25

3. Takahashi K, Tanabe K, Ohnuki M, Narita M, Ichisaka T, Tomoda K, Yamanaka S (2007) Induction of pluripotent stem cells from adult human fibroblasts by defined factors. Cell 131(5):861-872. https://doi.org/10.1016/j. cell.2007.11.019

4. Hu BY, Weick JP, Yu J, Ma LX, Zhang XQ, Thomson JA, Zhang SC (2010) Neural differentiation of human induced pluripotent stem cells follows developmental principles but with variable potency. Proc Natl Acad Sci U S A 107(9):4335-4340. https://doi.org/10.1073/ pnas.0910012107
5. Shi Y, Kirwan P, Livesey FJ (2012) Directed differentiation of human pluripotent stem cells to cerebral cortex neurons and neural networks. Nat Protoc 7(10):1836-1846. https://doi.org/ $10.1038 /$ nprot.2012.116

6. Centeno EGZ, Cimarosti H, Bithell A (2018) $2 \mathrm{D}$ versus $3 \mathrm{D}$ human induced pluripotent stem cell-derived cultures for neurodegenerative disease modelling. Mol Neurodegener 13(1):27. https://doi.org/10.1186/s13024-018-0258-4

7. Lancaster MA, Knoblich JA (2014) Generation of cerebral organoids from human pluripotent stem cells. Nat Protoc 9(10):2329-2340. https://doi.org/10.1038/nprot.2014.158

8. Lancaster MA, Renner M, Martin CA, Wenzel D, Bicknell LS, Hurles ME, Homfray T, Penninger JM, Jackson AP, Knoblich JA (2013) Cerebral organoids model human brain development and microcephaly. Nature 501(7467):373-379. https://doi.org/ 10.1038 /nature 12517

9. Gabriel E, Gopalakrishnan J (2017) Generation of iPSC-derived human brain organoids to model early neurodevelopmental disorders. J Vis Exp 122:55372. https://doi.org/10. $3791 / 55372$

Open Access This chapter is licensed under the terms of the Creative Commons Attribution 4.0 International License (http://creativecommons.org/licenses/by/4.0/), which permits use, sharing, adaptation, distribution and reproduction in any medium or format, as long as you give appropriate credit to the original author(s) and the source, provide a link to the Creative Commons license and indicate if changes were made.

The images or other third party material in this chapter are included in the chapter's Creative Commons license, unless indicated otherwise in a credit line to the material. If material is not included in the chapter's Creative Commons license and your intended use is not permitted by statutory regulation or exceeds the permitted use, you will need to obtain permission directly from the copyright holder. 